\title{
Keepered-Perpendicular-Media Reproduction with Dual MR Heads
}

\author{
L.N. He, Z.G. Wang, D.J. Mapps, D.T. Wilton, W.W.Clegg and P.Robinson \\ Centre for Research in Information Storage Technology, \\ School of Electronic, Communication and Electrical Engineering, University of Plymouth, Devon PL4 8AA, United Kingdom
}

\begin{abstract}
The key technique to apply keepered media system is how to "open" the keepered layer. In this paper, we would propose a new concept of keepered-media-reproduction with Dual MR heads. The keeper layer could be biased by the stray flux from DMR sensors. The shunting-releasing ratio between reproductive signals without and with sense current is found to be $\mathbf{- 2 6 . 6}$ dB. This is a completely-stable medium system against thermal demagnetization. It is believed that keepered media may offer a similar advantage with MR heads and potentially allow magnetics to break the $10 \mathrm{~Gb} / \mathrm{in}^{2}$ areal density barrier due to the high performance gains of MR heads.
\end{abstract}

\section{INTRODUCTION}

As recording densities exceed $10 \mathrm{~Gb} / \mathrm{in}^{2}$, recorded transitions may become unstable, causing magnetically recorded information to degrade over time. A top keepered layer adjacent to the hard-magnetic storage layer can profoundly affect the recorded magnetic transitions in the media. The keeper layer reduces the demagnetization of the magnetic transition and hence the transition length, smoothes zigzag transitions, and significantly reduces the effects of finite head-pole width on the output. As a result, keepering not only boosts recording densities, but has the potential to stabilize recorded data against thermal demagnetization - which may become a significant issue when recording densities approach $10 \mathrm{~Gb} / \mathrm{in}^{2}$. In a typical perpendicular magnetic recording system, a soft-magnetic underlayer is well used to enhance the read sensitivity. An improved perpendicular media design with both top and bottom soft-magnetic keepered layers is discussed here. The top softmagnetic keepered layer will further stabilize recorded data against thermal demagnetization and improve read characteristics.

The key technique to apply keepered media system is how to "open" the keepered layer. In other words, the keepered media system requires a small bias field during read operations. Work to date has been done with thin-film and metal-in-gap inductive heads together with keepered longitudinal media since it

Manuscript completed 10 July, 1997

Corresponding author: Dr. L.N.He is with School of Elect., Communication \& Elect. Eng., University of Plymouth, Drake Circus, Plymouth, Devon PL4 8AA, United Kingdom. FAX: +44-1752-232583, E-mail: lhe@plymouth.ac.uk

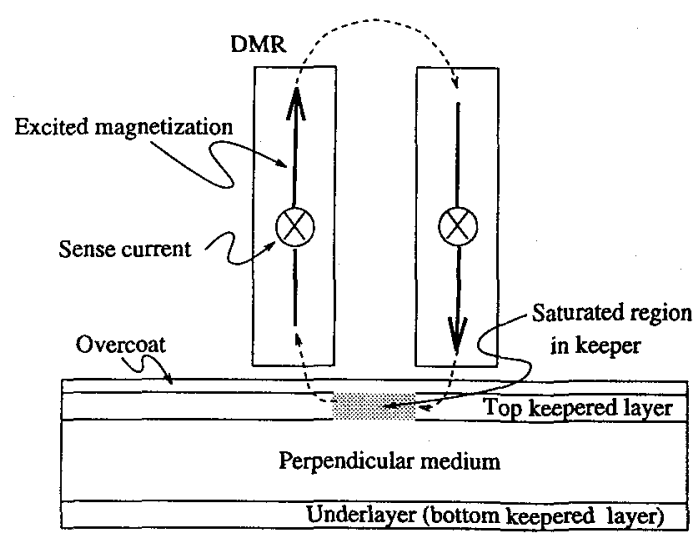

Fig. 1. Media cross-section and flux path for a basic keepered-perpendicularmedia reproduction with a Dual MR head. A soft-magnetic keeper film is deposited on top of the stronger layer and below the lubricating overcoat. During a write operation, flux from the recording head saturates the keeper above the stronger region. The keeper layer could be biased by the stray flux from dual MR sensors during a read operation.

is relatively easier to produce head-bias field by the ring head structure[1][2]. In this paper, we would propose a new concept of keepered-media reproduction with Dual MR heads. It is believed that keepered media may offer a similar advantage with MR heads due to the high performance gains of MR heads.

\section{PRINCIPLE}

The principle of keepered-media reproduction with Dual MR heads is illustrated in Fig.1. Media cross-section and flux path for a basic keepered-perpendicular-media reproduction with a Dual MR head are shown. A soft-magnetic top keeper film is deposited on the stronger layer and below the lubricating overcoat. During a write operation, flux from a submicrontrackwidth single-pole recording head[3](not shown in the figure) saturates the keeper above the stronger region. The keeper layer could be biased by the stray flux from dual MR sensors during a read operation.

Utilisation of two MR elements in a differential mode[4] is attractive and has led to different head designs: dual MR (DMR)[5][6], gradiometer[7], and dual stripe MR (DSMR)[8][9][10][11]. The 2-terminal DMR heads, consisting of two nominally identical, adjacent magnetoresistive 


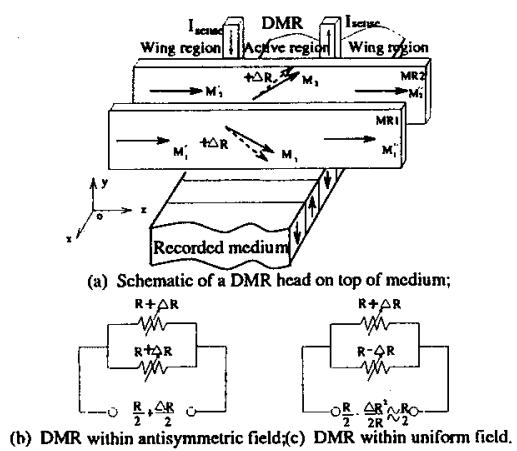

Fig. 2. Schematic of a Dual Magnetoresistive head. DMR is a typical 2 terminal element because the two MR stripes are shorted at both ends.

stripes separated by a dielectric spacer (e.g. $\mathrm{S}_{i} \mathrm{O}_{2}$ or $\mathrm{Al}_{2} \mathrm{O}_{3}$ ) in the active region but shorted at both ends (the contact regions underneath leads and off-track wing regions) by conducting tabs (e.g. $\mathrm{A}_{u}$, but might be replaced with conducting permanent magnet or exchange-coupled material) between MR stripes, is designed to combine the simpler fabrication with the larger intrinsic sensitivity at high linear density[6]. In DMR, the magnetizations of the individual stripes are biased to a semi-saturation state by the sense current passing through the opposite stripe, as shown in Fig.2(a). This means that the magnetizations scissor from their nominal parallel position, one is at $-45^{\circ}$, the other is at $+45^{\circ}$. The nominal parallel magnetization configuration without sense current flowing is caused by permanent magnet tabs (e.g. $\mathrm{C}_{o}$ alloy) or exchange-coupled tabs (e.g. $\mathrm{F}_{e} \mathrm{M}_{n}$ or $\mathrm{T}_{b} \mathrm{C}_{o}$ ) placed in the contact regions and wing regions to control the magnetization orientation thus avoiding multidomain formation within the soft films[12][13][14]. DMR is a typical 2-terminal element because the two MR stripes are shorted at both ends. In other words, these two MR stripes are connected in parallel electrically. In Fig.2 (a) and (b), the two MR stripes are exposed to the anti-symmetric field from a recorded medium bit and thus the DMR head produces an output(both these two MR stripes within an anti-symmetric field have resistance change with same change polarity). In Fig.2 (c), the two MR stripes are exposed to an uniform applied field and thus the DMR head produces almost null output(one MR stripe has resistance increase, the other has resistance decrease and they cancelled each other for parallel connection). In this regard, the DMR is sensitive only to the spatially anti-symmetric component of the applied field. Most importantly, this DMR heads promises excellent resolution when reading the high field gradients from extremely sharp magnetization transitions, e.g., thoses in good perpendicular media, due to the small separation of the elements in the device. These features are important for overcoming the limitations of present recording heads and achieving the high spatial resolution necessary for an ultra-high density perpendicular magnetic recording system[15][16][17].

\section{Finite ElEMENT MODEL}

The TOSCA 3-D FEM Nonlinear Electromagnetic Field Simulation Package [18] (supplied by Vector Field Corp.) is used to study the playback behaviour in keepered perpendicular media system. Finite element discretization forms the basis of the methods used in these analysis programs. These programs provide facilities for the creation of finite element models, specification of complicated conductor geometry, definition of material characteristics including for example, nonlinear and anisotropic descriptions and graphical displays for examination of the data.

Due to the symmetric structure of the heads, only half of the structure is needed for the simulation. The maximum element number used in the modelling was 45,000 . An open boundary was used in the model. The effect of the open boundary condition on the accuracy of the solution was verified by comparing the solutions obtained by applying the potential and derivative boundary conditions to the open boundary, and the errors between the two were within $3.5 \%$.

The output of the MR head was evaluated by the volume integral of magnetic flux density within the MR stripe. Its expression is as below:

$$
\text { Output of single } M R \text { stripe } \propto \iiint B y d x d y d z
$$

The volume integral's unit is $\mathrm{T} \cdot \mu \mathrm{m}^{3}$. During a read operation, we should add the integral over the right stripe and that over the left one as the final output of DMR head because within an anti-symmetric field both these two MR stripes, in parallel electrically in a DMR head, have resistance change with same change polarity.

\section{ANALYSIS}

The aim of a replay process simulation is to verify the feasibility of keepered-medium reproduction with DMR heads. After obtaining the volume integral (i.e. effective signal) of the DMR sensor in the presence of a typical perpendicular medium, we can compare the magnitude difference between the reproductive (or releasing) case and shunting case. In other words, we should give the proportion of the unexpected signal without sense current flowing within the effective reproductive signal with sense current flowing. In order to simplify the problem, only a two-bit perpendicular medium right underneath the read gap were simulated. The left bit is magnetized in the down direction and the right bit magnetized in the up direction. The trackwidth of the two-bit medium is $0.3 \mu \mathrm{m}$ [3]; the bit length is $0.2 \mu \mathrm{m}$ with no transition length; the thickness of the medium 
is $0.07 \mu \mathrm{m}$; the head-medium spacing is $0.012 \mu \mathrm{m}$; the coercivity of the perpendicular recording medium is $1650 \mathrm{Oe}$ with an MrT of 2.4 memu; the keeper layer thickness is nominally 200 $\AA$, with a coercivity of 15 Oe and a saturation magnetization of about 1.2 Tesla; the thickness of the keeper/record layer separation is $25 \AA$; the thickness of the $\mathrm{C}+$ lubricating layer is 25 $\AA$. The DMR head structure and dimensions used in the replay simulation are list below: the shield material is chosen to be a FeN based soft magnetic film with a saturation magnetization of 2.1 Tesla and an initial permeability of 3500 ; the read gap here is defined as the shield-to-shield separation, $0.35 \mu \mathrm{m}$; two parallel soft magnetic stripes in gap configuration have been used; both these stripes are assumed to be NiFe material for the two MR sensors; the MR track width is $0.28 \mu \mathrm{m}$; the MR stripe depth is $0.25 \mu \mathrm{m}$; the MR stripe thickness is $500 \AA$; the thicknesses of the dielectric layers are $1000 \AA, 500 \AA$ and 1000 $\AA$, respectively; the stripe-stripe separation, $500 \AA$, governs the linear resolution of readback processes.

In 2 terminal DMR heads, sense current of the same magnitude and direction is sent through both elements producing opposing bias fields in each sensor. The stray field distribution of the DMR in the presence of a top keeper layer (without recording layer) at a selected sense current $I=1.5 \mathrm{~mA}$ in the mid-width cut plane is shown in Fig.3. The field distribution is significantly up-down asymmetric due to the existence of the keeper layer. A strong field has been produced in front of the ring-head-like stripe-stripe gap. This figure also illustrates the magnetization depth distribution around the read gap region. The darkness is proportional to depth of magnetization saturation. In particular, from the figure, the resulting bias field in front of the stripe-stripe gap saturates a small region of the keeper layer. In a simple word, a "window" has been opened in the keeper layer. In contrast, the two MR stripes are only semi-saturated, i.e. $45^{\circ}$ biased, just suited for signal reproduction.

Figure 4 is a computed three dimensional flux distribution of the recorded bits in the mid-width cut plane, with the replay DMR head with a optimum sense current $I=1.5 \mathrm{~mA}$ at the top of the medium. The direction of each arrow corresponds to the direction of the field and its thickness to the field magnitude. From the figure, it is found that the small saturated region in the top keepered layer, which is created by the resulting bias field in front of the stripe-stripe-gap, cuts off the return path of the medium transition flux through the top keepered layer due to saturated region's low permeability. The medium transition flux is therefore forcibly released from the shunting in the keeper layer, which allows flux transitions to be picked up by the reproductive DMR head. For this reason, what Fig.4 shows is a "releasing state". The leakage flux from the medium goes upward in the right stripe and returns downward in the left stripe. Obviously the anti-symmetrically-distributed field components will cause a remarkable signal output for 2 stripes shorted at both ends in the DMR head. The calculated DMR

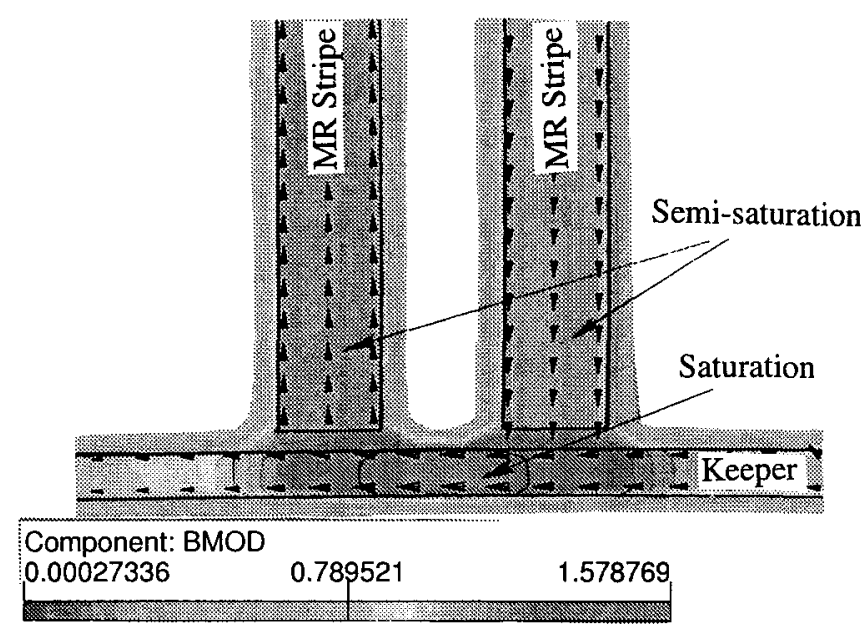

Fig. 3. The stray field distribution of the DMR in the presence of a top keeper layer (without recording layer) at a selected sense current $I=1.5 \mathrm{~mA}$. Sense current of the same magnitude and direction is sent through both elements producing opposing semi-saturated state in each sensor. The resulting bias field in front of the stripe-stripe-gap saturates a small region of the keeper layer underneath the gap.

output is $8.168 \times 10^{-19} \mathrm{~T} \cdot \mu \mathrm{m}^{3}$.

In contrast, we also computed three dimensional flux distribution in shunting state, without sense current flowing through the MR stripes. As shown in Fig.5, the flux distribution from the perpendicular recording layer is well restrained beneath the top keeper layer and above the bottom keeper layer(underlayer). In a similarly simple word, the "window" has been closed in the keeper layer. Almost null stray flux from the medium enters the MR stripes to be picked up by the reproductive DMR head. In this case, only $3.851 \times 10^{-20} \mathrm{~T} \cdot \mu \mathrm{m}^{3}$ is caused in the output of the DMR head. (This value is fictitious because a MR sensor can not work without sense current flowing through the stripes.)

In order to evaluate fairly the proportion of the contributions to the MR output between shunting case and releasing case, we define a Shunting-Releasing Ratio (SRR) as below:

$$
S R R=20 \log \frac{\text { Signal without sense current }}{\text { Signal with sense current }}(d B)
$$

In our investigation, the signal with sense current (releasing case) is $8.168 \times 10^{-19} \mathrm{~T} \cdot \mu \mathrm{m}^{3} / 3.851 \times 10^{-20} \mathrm{~T} \cdot \mu \mathrm{m}^{3}=$ 21.21 times as large as the signal without sense current (shunting case), so the SRR is $-26.6 \mathrm{~dB}$. Therefore the designed top keepered layer plays a reasonable role in shunting and releasing the medium transition flux. 


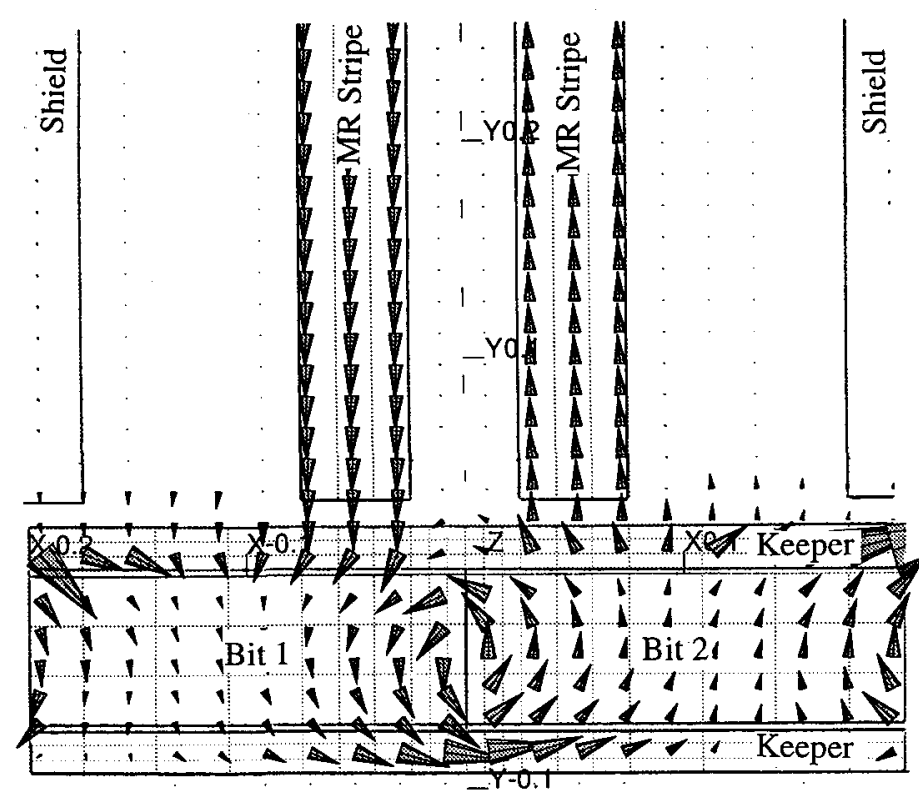

Fig. 4. Releasing state. Flux distribution of the recorded bits in the mid-width cut plane, with the replay DMR head with a optimum sense current $I=1.5$ $\mathrm{mA}$ at the top of the medium, is shown. The small saturated region in the top keepered layer, which is created by the resulting bias field in front of the stripe-stripe-gap, cuts off the return path of the medium transition flux through the top keepered layer due to saturated region's low permeability. The medium transition flux is therefore forcibly released from the shunting in the top keeper layer, which allows flux transitions to be picked up by the reproductive DMR head.

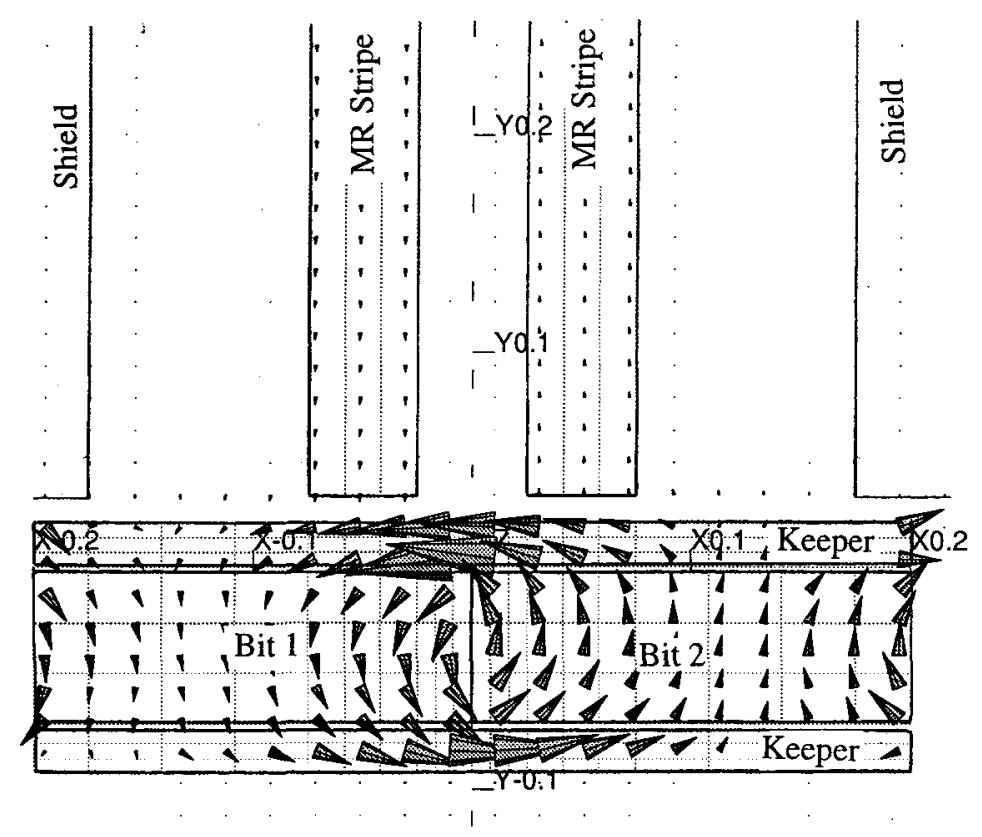

Fig. 5. Shunting state. Flux distribution of the recorded bits in the mid-width cut plane, with the replay DMR head without any sense current is shown. The flux distribution from the perpendicular recording layer is well restrained beneath the top keeper layer and above the bottom keeper layer(underlayer).

\section{CONCLUSIONS}

In this paper, we propose a new concept of keepered-mediareproduction with Dual MR heads. To verify its feasibility, a TOSCA 3-D FEM Nonlinear Electromagnetic Field Simulation Package is used. As a result, the keeper layer could be biased by the stray flux from DMR sensors. A so-called "shunting-releasing ratio" between reproductive signals without and with sense current is found to be $-26.6 \mathrm{~dB}$. This doublekeepered perpendicular medium is a completely-stable system against thermal demagnetization. It is believed that keepered media may offer an advantage with MR heads and potentially allow magnetics to break the $10 \mathrm{~Gb} / \mathrm{in}^{2}$ areal density barrier due to the high performance gains of MR heads. In addition, the principle stated here is also applicable to longitudinal media systems although only perpendicular medium has been included in this investigation.

\section{REFERENCES}

[1] B.Gooch, R.Niedemeyer, R.Wood and R.Pisharody, IEEE Trans., MAG-27, No.6, p.4549, 1991

[2] W.Reed, U.Cohen, D.Hollars, B.Zubeck, AB-10, Intermag'96

[3] Y.Nakamura, "A Challenge to Terabit Perpendicular Spinic Storage", J.Magn.Soc.Jpn, Vol.18, Supple.No.S1, pp161-170, 1994.

[4] D.J.Mapps, U.K. patent No. 2143071, 28/10/1987

[5] N.Smith, J.Freeman, P.Koeppe, and T.Carr, "Dual Magnetoresistive Head for Very High Density Recording", IEEE Trans. Magn., Vol. 28, No. 5, pp. 2292-2294, 1992.

[6] N.Smith, D.R.Smith, and S.Shtrikman, "Analysis of a Dual Magnetoresistive Head", IEEE Trans. Magn., Vol. 28, No. 5, pp. 2295-2297, 1992

[7] R.S.Indeck, J.H.Judy, and S.Iwasaki, "A Magnetoresistive Gradiometer", IEEE Trans. Magn., Vol. 24, No.6, pp. 2617-2619, 1988.

[8] Voegel, Patent US 3860965

[9] T.Authouy at al, IEEE Trans. Magn., Vol. 30, pp. 303, 1994

[10] Y. Hsu, C. C. Han, H. Chang, G. Ching, D. Hernandez, M. M. Chen, K. Ju, C. Che and J. Fitzpatrick, "Dual-Stripe MR Heads for One Gigabit per Inch Square Recording Density", INTERMAG'95, EB-01, San Antonio, 1995.

[11] Y.Guo, Y. Hsu, and K.Ju, "Recording Performance and Process Tolerance of Dual-Stripe MR Heads", IEEE Trans. Magn., Vol. 32, No. 1, pp. 31-37, 1996

[12] J.Zhu and D.J.O'Connor, "Impact of Microstrucure on Stability of Permanent Magnet Biased Magnetoresistive Heads", IEEE Trans. Magn., Vol. 32, No. 1, pp.54-60, 1996.

[13] F.B.Shelledy, J.L.Nix, "Magnetoresistive Heads for Magnetic Tape and Disk Recording", IEEE Trans. Magn., Vol.28, No.5, p.2283-2288, 1992.

[14] Y.Shen, W.Chen, W.Jensen, D.Ravipati, V.Retort, R.Rottmayer, S.Rudy, S.Tan and S.Yuan, "Comparative Study of MR Head Designs with Abutted vs. Overlaid Permanent Magnet Bias", IEEE Trans. Magn., Vol. 32, No. 1, pp. 19-24, 1996.

[15] Z.G. Wang, D.J. Mapps, D.T.Wilton, L.N.He, "Crossfeed problems in read-while-write inductive/GMR tape head", IEEE Trans. Magn., Vol.33, No.4, pp.2531-2537, July 1997.

[16] Z.G. Wang,L.N.He, D.J. Mapps, Y.Nakamura, W.W.Clegg, "Feasibility of Ultra-Dense Spin-Tunneling Random Access Memory (STram)", IEEE Trans. Magn., Vol.33, No.5, September 1997.

[17] Z.G. Wang, L.N. He, D.J. Mapps, Y. Nakamura, D.D. Clegg and D.T. Wilton, "Faster and Denser Spin-Tunneling Random Access Memory (STram)", IEEE Trans. Magn., Vol.33, No.5, September 1997.

[18] TOSCA Reference Manual, Vector Field Ltd., Oxford, United Kingdom, 1992. 